\title{
Hypoplasia of the Parabrachial/Kölliker-Fuse Complex in Perinatal Death
}

\author{
Anna Maria Lavezzi Giulia Ottaviani Lino Rossi Luigi Matturri \\ Institute of Pathology, University of Milan, Milan, Italy
}

\section{Key Words}

Parabrachial nuclei · Parabrachial/Kölliker-Fuse complex $\cdot$ Arcuate nucleus $\cdot$ Pulmonary hypoplasia

\begin{abstract}
We report the first observation of perinatal death attributable to morphological alterations of the parabrachial/ Kölliker-Fuse complex. In an infant, born at 41 weeks of gestation with severe signs of asphyxia and dead $20 \mathrm{~h}$ after delivery, we observed the presence of rare and immature neurons in the brain stem areas of both lateral and medial parabrachial nuclei and the absence of the characteristic neurons of the Kölliker-Fuse nucleus. Such hypoplasia was associated with severe hypoplasia of the arcuate nucleus and pulmonary hypoplasia.
\end{abstract}

Copyright $\odot 2004$ S. Karger AG, Basel

\section{Introduction}

Hypoplasia of the arcuate nucleus is often present both in sudden infant death syndrome (SIDS) and perinatal death (stillbirth after the 25th week of gestation and neonatal death) [1-3]. The lack of inflammatory infiltrates, hemorrhages, gliosis, and other reactive changes have made it possible to establish the congenital, primitive, and probably genetically based nature of this developmental defect, allowing a correlation to be made between SIDS and neonatal and late sudden fetal death.

Hypoplasia of the arcuate nucleus is a plausible functional morphological substrate in SIDS, characterizing a subgroup of subjects with a chemoreceptive defect [2]. In sudden intrauterine unexplained death, this anomaly cannot be considered the cause of death [3-6]. In fact, both the central and peripheral chemoreceptors are normally well developed in late pregnancy, though not functioning, to make sure they do not interfere with the intrauterine survival of the fetus. However, these same receptors promptly show their chemoreflexogenic function upon birth, as soon as the blood circulation shifts from maternal-fetal oxygenation to neonatal respiration. Thus, chemoreceptiveness, which is insignificant during fetal life, becomes vitally important during intra- and postpartum periods $[7,8]$.

During the final stages of fetal life, the chemoreceptors are normally well developed and potentially functioning $[9,10]$. However, their stimulatory effect on respiration is nullified by the dominant inhibitory input from nervous structures located in the upper portion of the brain stem, in particular in the Kölliker-Fuse nucleus [4, 11-13]. During fetal life, therefore, the lungs do not appear to play any

\section{KARGER}

Fax +41613061234

E-Mail karger@karger.ch

www. karger.com
(C) 2004 S. Karger AG, Basel

0006-3126/04/0862-0092\$21.00/0

Accessible online at:

www. karger.com/bon
Luigi Matturri, MD, PhD

Institute of Pathology, University of Milan

Via della Commenda, 19

IT-20122 Milan (Italy)

Tel. +3902 5419521, Fax +3902 5419522, E-Mail luigi.matturri@unimi.it 
part in gas exchange, although episodic rapid and irregular respiratory movements have been found in experimental studies [14].

In the human fetus, periodic respiratory activity can be detected by ultrasound from the 11th week of gestation, and this becomes more marked as pregnancy progresses [9]. This fetal breathing regulates the release of tracheal fluid in the lung and consequently regulates alveolar expansion and seems to favor lung development [10]. Lack of respiratory movements in the fetus could, therefore, result in impaired development of the alveoli and in pulmonary hypoplasia $[1,15]$.

The reflexogenic center which inhibits chemoreflexogenesis is located in a complex structure of the dorsolateral rostral portion of the pons. It is made up of three nuclei: lateral parabrachial nucleus, medial parabrachial nucleus, and Kölliker-Fuse nucleus (parabrachial/Kölliker-Fuse complex) [4, 11-13].

To the best of our knowledge, the herein described case is the first reported observation of perinatal death attributable to morphological alterations of the parabrachial/ Kölliker-Fuse nuclei, associated with severe hypoplasia of the arcuate nucleus and pulmonary hypoplasia.

\section{Case Report}

A male neonate born at $41+1$ weeks of gestation after a normal pregnancy by cesarean section from a primipara mother was well developed, but showed serious signs of asphyxia. Labor was induced because of oligoamnios and prolonged pregnancy. An urgent cesarean delivery was performed due to sudden fetal cardiac arrest at fetal heart tracing. The Apgar scores were 1-3-3 with an umbilical arterial cord $\mathrm{pH}<7.00$. Immediate cardiopulmonary resuscitation was performed by external cardiac massage and assisted ventilation. After $2 \mathrm{~min}$, the heart resumed beating. Mechanical ventilation was initiated, using mask and bag. The response was poor, and an endotracheal tube was inserted and mechanical ventilation continued. The baby manifested tachycardia, hyperthermia, neurological disturbances including tonicoclonic movements, and, finally, deep coma with areflexia. The baby was pronounced dead $20 \mathrm{~h}$ after delivery, and a 3rd-degree hypoxic-ischemic encephalopathy was diagnosed.

The mother, a 30-year-old woman, had an unremarkable pregnancy, except for a mild hypothyroidism which required no therapy. There was no significant family history relevant to the case.

A complete postmortem examination was performed, including a systematic gross and microscopic evaluation of the body, the placental disk, the umbilical cord, and the membranes. All organs were fixed in $10 \%$ phosphate-buffered formalin, processed, and embedded in paraffin. The sections $(5 \mu \mathrm{m})$ were stained with hematoxylin and eosin and Heidenhain's azan.

Regarding the lung examination, the stage of development was evaluated on the basis of a macroscopic criterion used at autopsy, namely the correlation between lung weight and body weight (LW/ $\mathrm{BW}$ ), and according to microscopic criteria which are the presence of cartilaginous bronchi up to the distal peripheral level and the radial alveolar count. This parameter involves the evaluation, in at least ten histological fields (magnification $\times 40$ ), of the number of alveoli transected by a perpendicular line drawn from the center of the most peripheral bronchiole (recognizable by not being completely covered by epithelium) to the pleura or the nearest interlobular septum. The normal reference values for the last 3 months of gestation correspond to $>0.022$ for $\mathrm{LW} / \mathrm{BW}$, and for radial alveolar count they range from 2.2 to $4.4[16]$.

The brain stem was examined according to the protocol routinely followed by our institution, available on the web site http://users.unimi.it/ pathol/sids/tecnica_e.html. In particular, the brain stem, including cranially the lower third of the midbrain and caudally the medulla oblongata sectioned some millimeters distally from the lower pole of the inferior olivary nucleus, was entirely processed and serially cut. The pertinent nuclei in the histological serial sections were outlined, namely the parabrachial/Kölliker-Fuse complex, the gigantocellular nucleus, the hypoglossal nucleus, the dorsal vagus motor nucleus, the tractus solitarii nucleus, the nucleus ambiguus, the trigeminal tractus and nucleus, the arcuate nucleus, the reticular formation, and the principal inferior olivary nucleus. Plates in the atlas of Olszewski and Baxter were used for reference [17].

\section{Results}

At autopsy, the newborn was described as well developed, with birth weight $(3 \mathrm{~kg})$ and length $(47 \mathrm{~cm})$ corresponding to gestational age. The external examination was normal for gestational age and sex. Macroscopic examination did not reveal malformations or organ malposition. The placenta showed a normal developmental morphology with microscopic evidence of multifocal vascular abnormalities of mild degree (villous infarctions, fibrin aggregates). Histological analysis revealed a marked lymphocyte depletion of the thymus and massive vascular congestion of all organs.

The lungs presented subpleural petechiae. The left lung weighed $32.2 \mathrm{~g}$ and the right lung $26.5 \mathrm{~g}$ (normal LW $65 \pm$ $17 \mathrm{~g}$. Microscopic examination disclosed pulmonary hypoplasia with a poor alveolar distension (radial alveolar count index 1.8) and presence of cartilaginous bronchi up to the distal peripheral level. The pulmonary hypoplasia was confirmed by the LW/BW macroscopic index (0.01).

The brain weighed $426 \mathrm{~g}$ (normal $401 \pm 41 \mathrm{~g}$ ) and presented generalized vascular congestion. Dissection of the brain showed a congested hemorrhagic aspect of the choroid plexuses. No parenchymatous hemorrhages were detected. Histological examination disclosed hyperchromic nuclei in the thalamus and some neurons with cytoplasmic eosinophilia in the hippocampus.

Detailed examination of the brain stem showed severe hypoplasia of the arcuate nucleus which was absent 


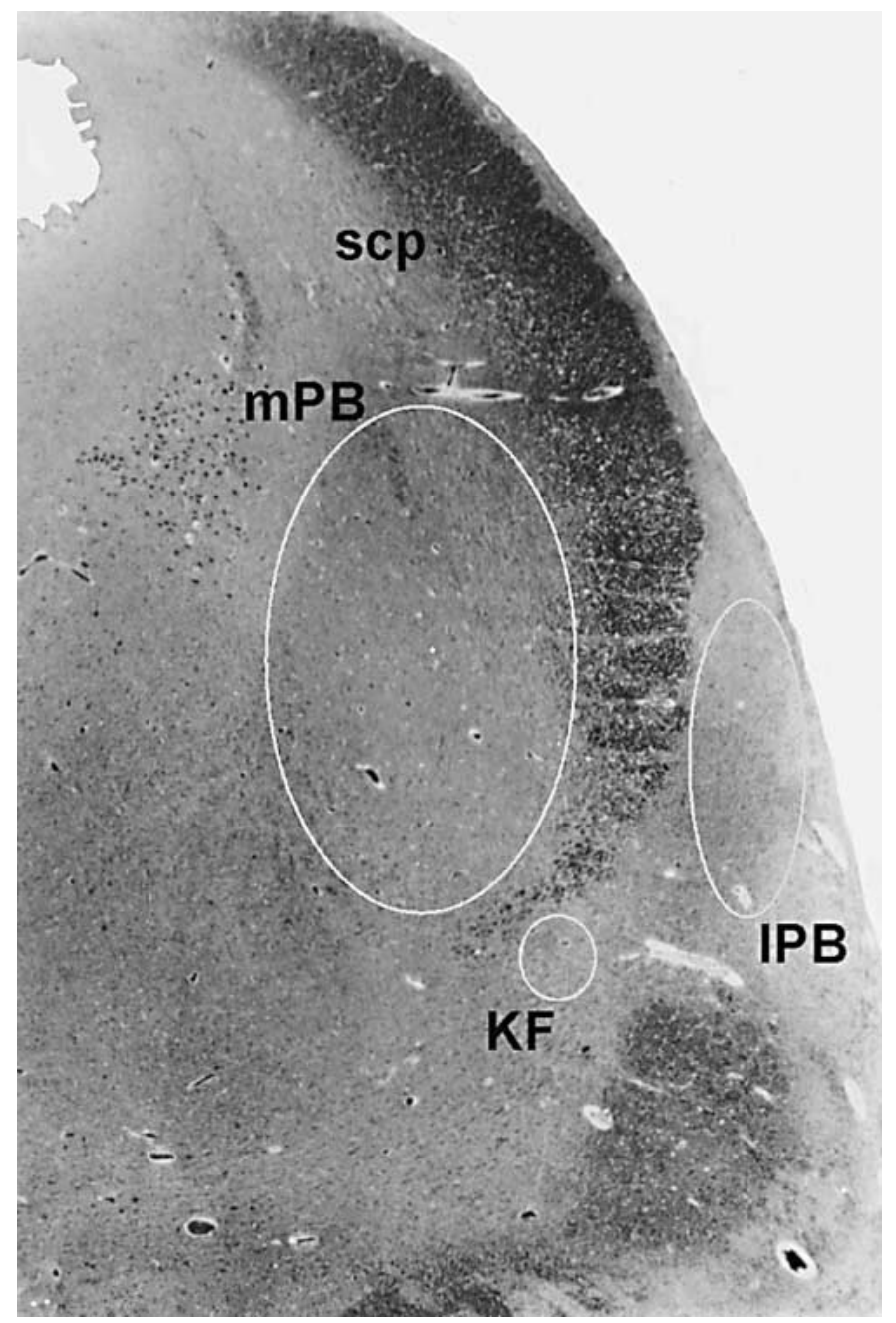

Fig. 1. Photomicrograph of a transverse hemisection of the rostral pons, showing in the encircled areas the location of the parabrachial/ Kölliker-Fuse complex. mPB = Medial parabrachial nucleus; $1 \mathrm{~PB}=$ lateral parabrachial nucleus; KF= Kölliker-Fuse nucleus; $\mathrm{scp}=$ superior cerebellar peduncle. Klüver-Barrera stain. $\times 20$.

throughout the entire rostromedial sections of the medulla oblongata and was only partially present at a caudal level, with neuronal immaturity. Hypoplasia of the reticular formation was also evident.

In the rostral transverse sections of the pons, only a few immature neurons were observed in either medial or lateral parabrachial nuclei, while the Kölliker-Fuse nucleus was not detectable, altogether conditioning the diagnosis of severe hypoplasia of the parabrachial/Kölliker-Fuse complex. Figure 1 represents a transverse hemisection of the human dorsolateral rostral pons, showing the location

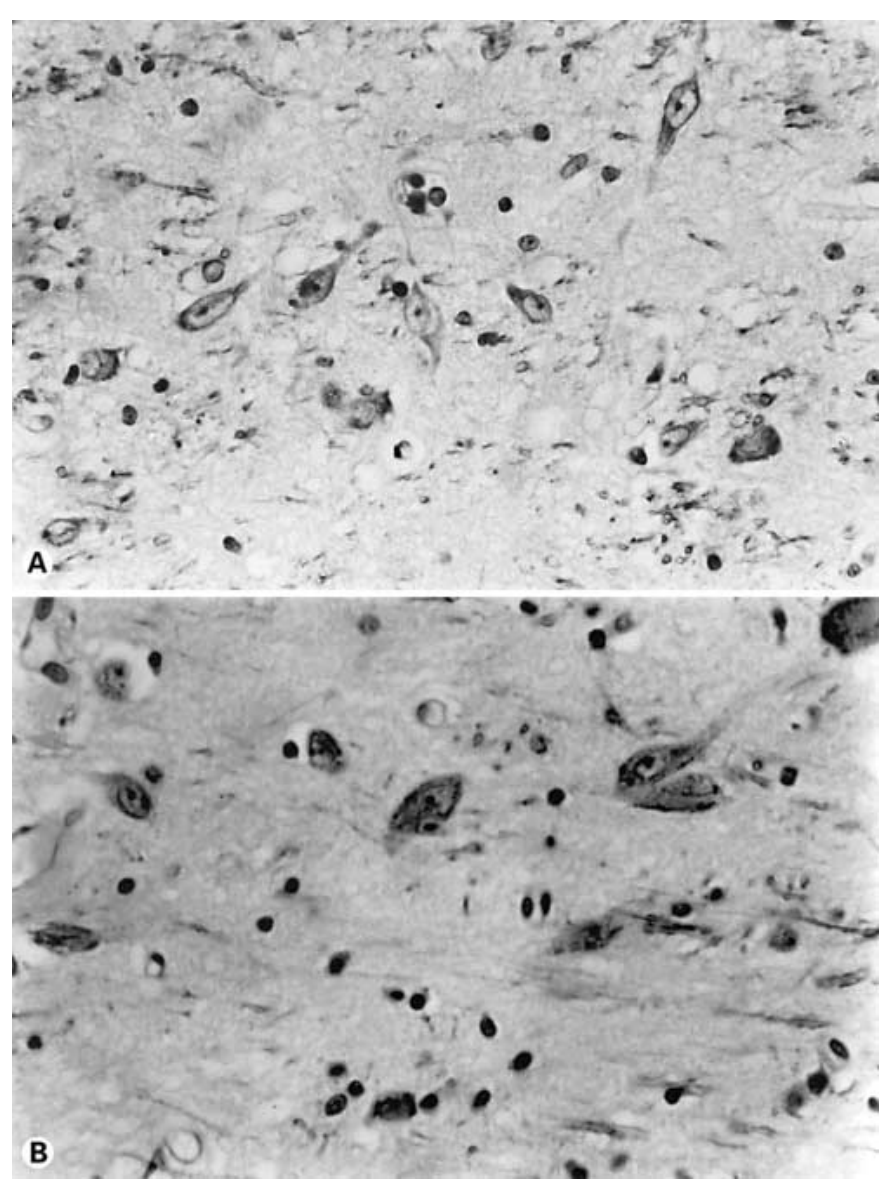

Fig. 2. Neurons of the lateral parabrachial nucleus in the reported case (A; male neonate born at $41+1$ weeks with severe hypoplasia of the parabrachial/Kölliker-Fuse complex) and in a control case (B; male neonate dead at 40 weeks of gestation from causes unrelated to abnormal central respiratory control, with a normal structure of the parabrachial/Kölliker-Fuse complex). Klüver-Barrera stain. $\times 50$.

of the two parabrachial nuclei and of the Kölliker-Fuse nucleus. Figures 2-4 show the neurons of the three complex nuclei observed in the reported case as compared with those of a control subject dead at 40 weeks of gestation from causes unrelated to abnormal central respiratory control, with a normal structure of the parabrachial/ Kölliker-Fuse complex. 


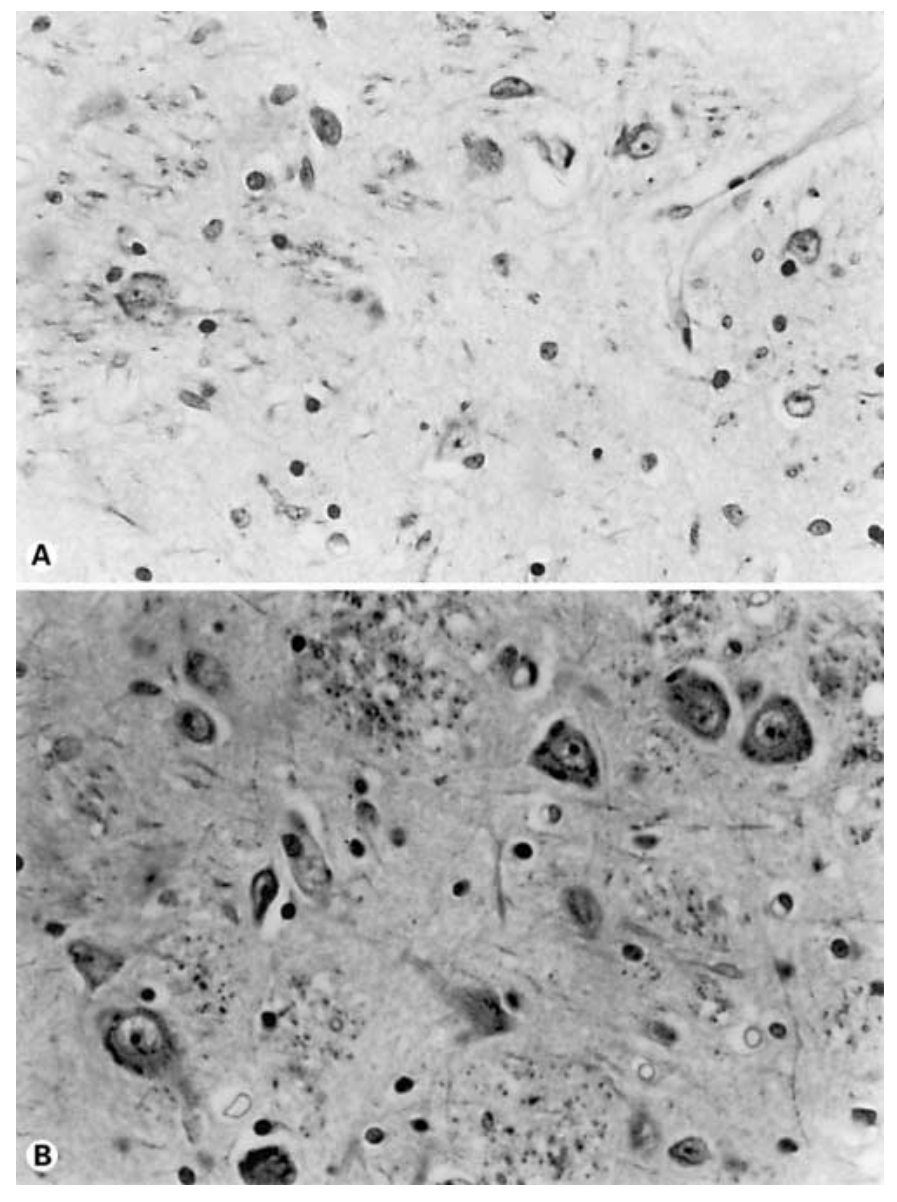

Fig. 3. Neurons of the medial parabrachial nucleus. A Reported case. B Control case described in the figure 2 legend. Klüver-Barrera stain. $\times 50$.

\section{Discussion}

It has been demonstrated in experimental studies that the parabrachial pontine nuclei and the Kölliker-Fuse nucleus play an essential role in the modulation of the respiratory activity, presenting with reciprocal afferent and efferent connections with the bulbar respiratory centers $[10-13,18]$. This complex has an important physiological function during intrauterine life, in that it inhibits the responses of peripheral and central chemoreceptors (already fully developed and potentially functional), therefore, suppressing any continuous active respiratory reflex [4].

Parabrachial/Kölliker-Fuse Hypoplasia

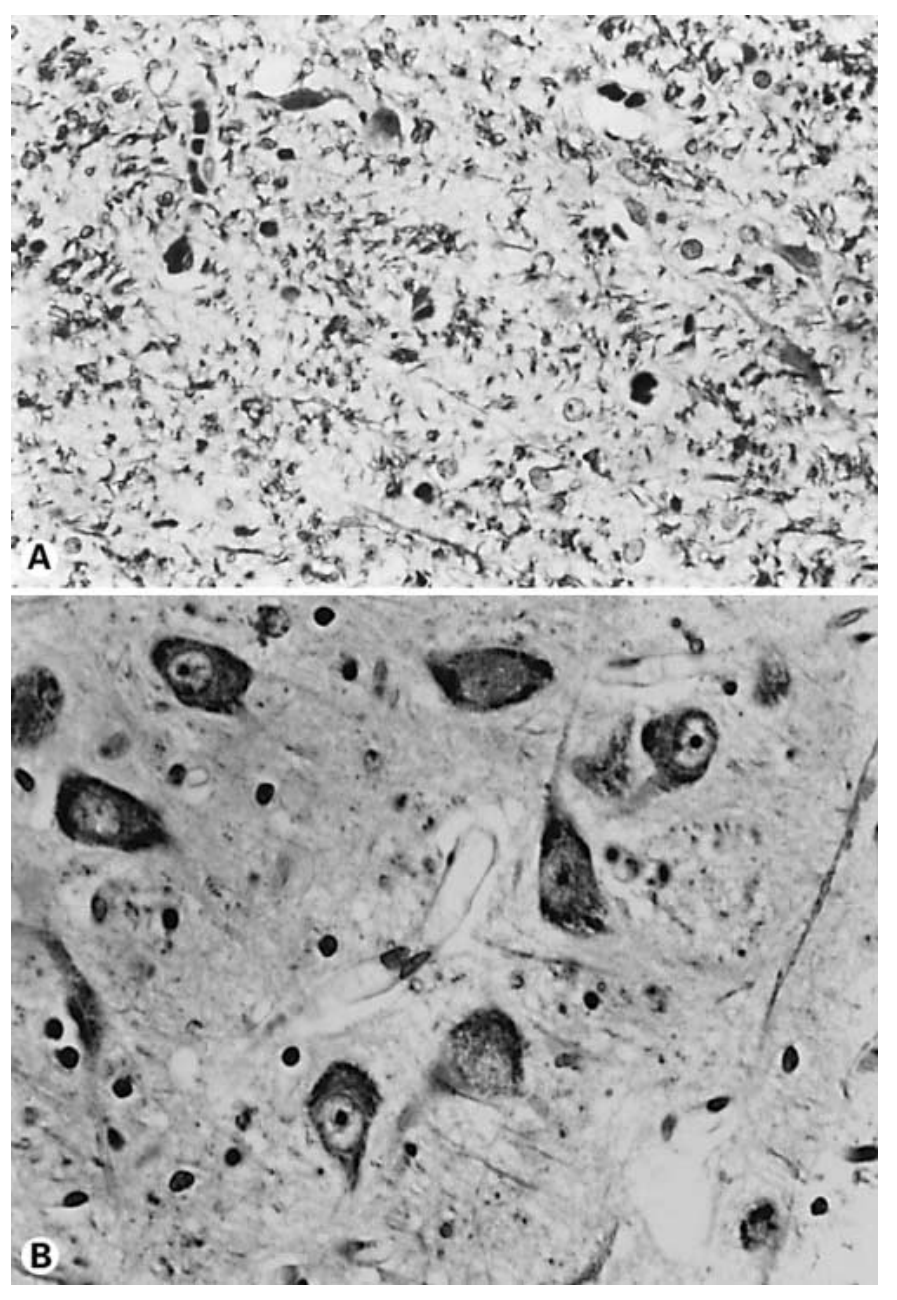

Fig. 4. Kölliker-Fuse nucleus. A Reported case. B Control case described in the figure 2 legend. In $\mathbf{A}$, the typical neurons of the Kölliker-Fuse nucleus are absent, while they are well detectable in $\mathbf{B}$. Klüver-Barrera stain. $\times 50$.

However, from time to time this inhibitory activity may be stopped to favor lung development $[9,10]$. These studies have shown that immediately after birth, the modulation of respiratory function of the parabrachial/Kölliker-Fuse complex is initiated. Kölliker-Fuse nucleus and medial parabrachial complex inhibit the neurons of the medulla oblongata from performing their role in inspiration and induce expiration, while the lateral parabrachial complex would stimulate, in turn, inspiration.

Detailed research on the parabrachial complex in the rat, based on retrograde and anterograde transport of specific agglutinins, allowed the definition not only of the physiological role, but also of the precise structure of the 
three nuclei [19-21]. In man, the impossibility of experimental approaches makes it difficult to functionally and morphologically characterize these structures. The results of the few studies performed in this field are imprecise and contradictory $[22,23]$.

In our very recent studies [4, 24], based on the detailed examination of serial sections of a large number of brain stems, we identified and outlined the cytoarchitecture of these three complex nuclei. The lateral parabrachial nucleus is located in between the lateral limit of the superior cerebellar peduncle and the lateral lemniscus. It extends vertically from the pons-mesencephalon junction (cranial pole) to the level, where the lateral lemniscus nucleus is clearly visible (caudal pole). It contains large round or tapering neurons with a light nucleus, a prominent nucleolus, and scarce cytoplasm.

The medial parabrachial nucleus is located in transverse sections, medially to the superior cerebellar peduncle, between motor nucleus of trigeminal nerve, locus coeruleus, and the top of the peduncle itself. Longitudinally, its size increases from the rostral pole (pons-mesencephalon junction) to the caudal pole (where the lateral lemniscus nucleus is clearly visible). A large number of neurons are present, larger than those of the lateral parabrachial nucleus and oval or polygonal in shape, with abundant cytoplasm.

The Kölliker-Fuse nucleus extends from the rostral section of the pons to the entire of the inferior portion of the mesencephalon. In transverse sections, especially, at the level of the caudal pole, it shows regrouped neurons located ventrally to the lateral parabrachial nucleus, in between the medial limit of the superior cerebellar peduncle and the medial lemniscus. These neurons, much larger than those of the parabrachial nuclei, are characterized by a large, eccentric nucleus, with evident nucleolus and Nissl substance adhering to the cell membrane. These neurons are partly densely regrouped (subnucleus compactus) and partly dispersed (subnucleus dissipatus).

In this case, the presence of rare and immature neurons in the areas of both the lateral and the medial parabrachial nuclei and the total absence of the characteristic neurons of the Kölliker-Fuse nucleus (fig. 4) led to the diagnosis of hypoplasia of the parabrachial/Kölliker-Fuse complex.

The presence of severe asphyxia at birth seems to be primarily attributable to the poor development of this pontine reflexogenic center, eventually destined to modulate the postnatal respiratory activity. This was also concomitant with the marked hypoplasia of the arcuate nucleus, one of the main bulbar centers involved in respiratory neuronal circuitry for reflexogenic $\mathrm{pCO}_{2} / \mathrm{pO}_{2} / \mathrm{pH}$ responsiveness $[2,5]$, complicated by pulmonary hypoplasia [1].

The developmental defect of the parabrachial/Kölliker-Fuse complex did not, however, prevent pregnancy from reaching term. The presumed failure of these nuclei to exert their normal inhibitory effect on chemoreflexogenesis during the prenatal period was likely accompanied in this case by the absence of sporadic fetal respiratory movements needed for the alveoli to mature [10], as confirmed by the occurrence of pulmonary hypoplasia [1, $10]$.

\section{Acknowledgments}

This study was supported by the Lombardy Region (project No. 49210-24.3.00 'Programma di ricerche e interventi per la riduzione del rischio di morte improvvisa del lattante - SIDS - e di morte inaspettata del feto') and MURST Cofin 2000 (project No. MM06153578 'Pathologic study of late fetal unexplained death-stillbirth').

\section{References}

1 Matturri L, Lavezzi AM, Minoli I, Ottaviani G, Rubino B, Cappellini A, Rossi L: Association between pulmonary hypoplasia and hypoplasia of arcuate nucleus in stillbirth. J Perinatol 2003;23:328-332.

2 Matturri L, Ottaviani G, Alfonsi G, Crippa M, Rossi L, Lavezzi AM: Study of the brainstem, particularly the arcuate nucleus, in sudden infant death syndrome (SIDS) and sudden intrauterine unexplained death (SIUD). Am J Forensic Med Pathol 2004;25:44-48.
3 Matturri L, Minoli I, Lavezzi AM, Cappellini A, Ramos S, Rossi L: Hypoplasia of medullary arcuate nucleus in unexpected late fetal death (stillborn infants): A pathologic study. Pediatrics 2002; 109:E43.

4 Lavezzi AM, Ottaviani G, Ballabio G, Rossi L, Matturri L: Preliminary study on the cytoarchitecture of the human parabrachial/KöllikerFuse complex, with reference to sudden infant death syndrome (SIDS) and sudden intrauterine unexplained death (SIUD). Pediatr Dev Pathol 2004, in press (publ online March 17, 2004).
5 Filiano J, Kinney HC: Arcuate nucleus hypoplasia in the sudden infant death syndrome. $\mathrm{J}$ Neuropathol Exp Neurol 1992;51:394-403.

6 Froen JF, Arnestad M, Frey K, Vege A, Saugstad OD, Stray-Pedersen B: Risk factors for sudden intrauterine unexplained death: Epidemiologic characteristics of singleton cases in Oslo, Norway, 1986-1995. Am J Obstet Gynecol 2001;184:694-702.

7 Cohen M: Neurogenesis of respiratory rhythm in the mammal. Physiol Rev 1979;59:91-104. 
8 Feldman JL: Neurophysiology of breathing in mammals; in Bloom FE (ed): Handbook of Physiology, section 1: The Nervous System. Intrinsic Regulatory System of the Brain. Bethesda, American Physiological Society, 1986, vol IV, pp 463-524.

9 Boddy K, Dawes GS: Fetal breathing. Br Med Bull 1975;31:3-7.

10 Harding R, Bocking AD, Sigger JN: Influence of upper respiratory tract on liquid flow to and from fetal lungs. J Appl Physiol 1986;61:6871.

11 Walker DW: Hypoxic inhibition of breathing and motor activity in the foetus and newborn. Clin Exp Pharmacol Physiol 1995;22:533536.

12 Younes M, Baker J, Remmers JE: Temporal changes in effectiveness of an inspiratory inhibitory electrical pontine stimulus. J Appl Physiol 1987;62:1502-1512.

13 Holmes HR, Herbert H, Saper CB: Parabrachial subnuclei in respiratory control. Soc Neurosci 1987;13:1686.
14 Bystrzycka EB, Nail BS, Purves MJ: Central and peripheral neural respiratory activity in the mature sheep foetus and newborn lamb. Respir Physiol 1975,25:199-215.

15 Barness EG: Respiratory system; in Barness EG (ed): Potter's Pathology of the Fetus and Infant. St. Louis, Mosby, 1997, pp 712-773.

16 Askenazi SS, Perlman M: Pulmonary hypoplasia: Lung weight and radial alveolar count as criteria of diagnosis. Arch Dis Child 1979;54: 614-618.

17 Olszewski J, Baxter D: Cytoarchitecture of the Human Brain Stem. Basel, Karger, 1982.

18 Segers LS, Shannon R, Lindsey BG: Interactions between rostral pontine and ventral medullary respiratory neurons. J Neurophysiol $1985 \cdot 54: 318-334$
19 Herbert H, Moga MM, Saper CB: Connections of the parabrachial nucleus with the nucleus of the solitary tract and the medullary reticular formation in the rat. J Comp Neurol 1990;293: 540-580.

20 Fulwiler CE, Saper CB: Subnuclear organization of the efferent connections of the parabrachial nucleus in the rat. Brain Res 1984;319: 229-259.

21 Granata A: Ascending and descending convergent inputs to neurons in the nucleus parabrachialis of the rat: An intercellular study. Brain Res 1993;600:315-321.

22 Gioia M, Rodella L, Petruccioli MG, Bianchi $\mathrm{R}$ : The cytoarchitecture of the adult human parabrachial nucleus: A Nissl and Golgi study. Arch Histol Cytol 2000;63:411-424.

23 Block CH, Estes M: The cytoarchitectural organization of the human parabrachial nuclear complex. Brain Res Bull 1990;24:617-626.

24 Lavezzi AM, Ottaviani G, Rossi L, Matturri L: Cytoarchitectural organization of the parabrachial/Kölliker-Fuse complex in man. Brain Dev, in press. 